\title{
Genetic engineering of medium-chain-length fatty acid synthesis in Dunaliella tertiolecta for improved biodiesel production
}

\author{
Huixin Lin ${ }^{1}$ - Yuan Kun Lee ${ }^{1}$ \\ Received: 17 April 2017 /Revised and accepted: 22 June 2017 /Published online: 15 July 2017 \\ (C) The Author(s) 2017. This article is an open access publication
}

\begin{abstract}
Genetic engineering of microalgae to accumulate high levels of medium-chain-length fatty acids (MCFAs) represents an attractive strategy to improve the quality of microalgae-based biodiesel, but it has thus far been least successful. We demonstrate that one limitation is the availability of fatty acyl-acyl carrier protein (ACP) substrate pool for acylACP thioesterase (TE). A combinational expression platform that involved plant lauric acid-biased TE (C12TE) and MCFA-specific ketoacyl-ACP synthase (KASIV) increased lauric acid (C12:0) and myristic acid (C14:0) accumulation by almost sevenfold and fourfold, respectively, compared with native strain. These findings suggest a platform for further investigation into the enlargement of MCFA acyl-ACP substrate pool as an approach to sustainably improve quality of microalgae-based biodiesel with regard to MCFA production.
\end{abstract}

Keywords Microalgae $\cdot$ Thioesterase $\cdot$ Ketoacyl-ACP synthase $\cdot$ Lipid $\cdot$ Fatty acid

\section{Introduction}

In response to the world energy crisis and the global climate change, microalgae-based biodiesel has attracted renewed interest in an effort to search for sustainable development (Zinoviev et al. 2010). While most of the contemporary microalgae-based biodiesel research efforts focus on improving biomass accumulation and lipid productivity, relatively

Yuan Kun Lee

micleeyk@nus.edu.sg

1 Department of Microbiology and Immunology, Yong Loo Lin School of Medicine, National University of Singapore, Block MD4, 5 Science Drive 2, Singapore 117545, Singapore few studies have investigated the quality of microalgae oil. The fuel performance properties of the biodiesel are directly dependent on the structural features of the biologically derived fatty acid (FA) profiles. Microalgal oils typically contain longer FA carbon chain lengths and higher degrees of unsaturation compared to regular petroleum diesel (Stansell et al. 2012). These biomass feedstock traits lead to poor cold flow temperature properties (longer FA carbon chain lengths) and oxidative instability (higher degrees of unsaturation) (Knothe et al. 2005).

Genetic engineering of FA profiles in microalgae to synthesize medium-chain-length FAs (MCFAs), from C8 to C14, is preferable for production of biodiesel (Durrett et al. 2008). The chain lengths of FAs are determined by acyl-acyl carrier protein (ACP) thioesterases (TEs). TEs are highly specific and are classified according to their substrate preferences, namely FatA and FatB. FatA class enzymes prefer C18:1-ACP as a substrate, whereas FatB class enzymes prefer saturated acylACPs (Jing et al. 2011). Certain plant species accumulate MCFAs in their seed oil storage, and accordingly, there exist chain-length specific TEs that prematurely cleave the corresponding FAs from the growing fatty acyl-ACPs (Voelker and Kinney 2001). For example, Umbellularia californica (Davies et al. 1991) and Cuphea hookeriana (Dehesh et al. 1996) seeds accumulate up to $90 \%$ MCFAs in the triacylglycerols (TAGs). The chain-length specific TEs were isolated and identified in both species as the cause of the unusual accumulation (Thelen and Ohlrogge 2002). The first proof of principle of introducing chain-length specific TE to alter FA profile was demonstrated by Voelker and colleagues (1992) via engineering a lauric acid (C12:0)-biased FatB TE from U. californica into Arabidopsis thaliana for a $24 \%$ increase in C12:0 production.

Divergent TEs with substrate specificities for FAs with chain lengths less than $\mathrm{C} 16$ were previously engineered to 
alter FA contents in microalgae. C12:0-biased FatB TE from U. californica and myristic acid (C14:0)-biased FatB TE from Cinnamomum camphora were transformed into Phaeodactylum tricornutum, which redirected FA synthesis to, albeit at low yield (6\% increase in C12:0 and $15 \%$ increase in C14:0), the desired medium-chain-length phenotype (Radakovits et al. 2011). Similar plant TEs were introduced into Chlamydomonas reinhardtii, but no change in FA profile was observed (Blatti et al. 2012). Distinct TEs in microalgae (C. reinhardtii and P. tricornutum) have been identified, and they are plant FatA/FatB hybrid TEs, demonstrating promiscuity in FA substrates they act upon (Gong et al. 2011; Blatti et al. 2012). Overexpression of the native $C$. reinhardtii TE resulted in a corresponding phenotype of enhanced MCFA production (Blatti et al. 2012). On the other hand, overexpression of P. tricornutum TE did not result in altered FA composition in P. tricornutum (Gong et al. 2011).

Past efforts to increase MCFA production in microalgae by genetic modifications of chain-length specific TEs have met with limited success. We are interested to investigate a less considered mechanism that may limit MCFA accumulation, and which is the availability of fatty acyl-ACP substrate pool for the TE activity. Such a mechanism may be related to the intrinsic competitive nature of endogenous TE whereby broadly specific microalgae TE could outrival chain-length specific exogenous TE in the hydrolytic activity of fatty acyl-ACPs. This control is likely related to the functions of lipids in the structural membrane compositions of unicellular microalgae, with primarily long-chain-length FAs (LCFAs), from C16 to C18 (Tang et al. 2011). Although TEs are necessary determinants of MCFA phenotype, other candidates for chain-length regulation activities may exist and they are ketoacyl-ACP synthases (KASs), the condensing enzymes responsible for the cyclic two carbon elongations in FA synthesis (Dehesh 2001). We speculate that a condensing enzyme has the ability to proportionate different chain lengths of fatty acyl-ACP substrates for the hydrolysis by TEs. In this study, we describe the results of various genetic modification approaches to increase MCFA production in Dunaliella tertiolecta. Our results additionally address whether endogenous TE and/or MCFA-specific KAS are involved in MCFA accumulation.

\section{Materials and methods}

\section{Microalgae strains and culture conditions}

Strain LB-999 of $D$. tertiolecta was obtained from the UTEX Culture Collection of Algae (University of Texas, Austin, TX, USA). Dunaliella tertiolecta cells were grown in a batch system in a sterile ATCC-1174 liquid medium (American Type Culture Collection, Manassas, VA, USA) containing 0.5 M
$\mathrm{NaCl}$ on a rotary shaker at $25^{\circ} \mathrm{C}$, under a $14 \mathrm{~h}$ light $/ 10 \mathrm{~h}$ dark regime $\left(50 \mu \mathrm{mol}\right.$ photons $\left.\mathrm{m}^{-2} \mathrm{~s}^{-1}\right)$. Cell densities were measured using an automatic cell counter (Bio-Rad Laboratories, USA). For the purpose of clarity, all experiments were performed in independent biological/technical triplicates and began at equal cell densities for standardization unless otherwise stated.

\section{Plasmid construction}

The plasmids used in this study are listed in Fig. 1. All of the TEs and KAS were synthesized and codon optimized for the expression in D. tertiolecta (GenScript Corporation, USA). The TE sequences used in this study were derived from a lauric acid-biased TE (C12TE) from U. californica (GenBank U17097.1) and a myristic acid-biased TE (C14TE) from C. camphora (GenBank U31813.1) (Radakovits et al. 2011; Blatti et al. 2012). The MCFAspecific KAS (KASIV) was derived from C. hookeriana (GenBank AAC68861.1) (Dehesh et al. 1998). Each gene, including a chloroplast-targeting sequence from D. tertiolecta sedoheptulose-bisphosphatase (DtSBPase) (GenBank KF193066), was cloned into a pGreenII 0000 plasmid (Hellens et al. 2000) that contained either zeocin (ble) (Stevens et al. 1996) or norflurazon (DsPDS-L502F) (Liu et al. 2013) conferring resistance genes, under the control of D. tertiolecta ribulose bisphosphate carboxylase small subunit 1 (DtrbcS1) promoter (Walker et al. 2005). The overexpression plasmids were abbreviated as pPrbcS-cC12TE-ble, pPrbcS-cC14TE-ble, and pPrbcS-KASIV-PDSF. A 300-bp fragment of $D$. tertiolecta TE (DtTE) (GenBank KX815261) was used to construct the DtTE knockdown plasmid, pDtTERNAi, driven by the DtrbcS1 promoter, according to our previous study (Lin et al. 2013).

\section{Microalgae transformation}

pPrbcS-cC12TE-ble, pPrbcS-cC14TE-ble, pDtTE-RNAi, and pPrbcS-KASIV-PDSF were transformed into wild-type and mutant cC12TE_A4 (from UcC12TE background) D. tertiolecta (Fig. 1) using the glass beads method (Lin et al. 2013). Selection of transgenic $D$. tertiolecta strains was done in either $8 \mu \mathrm{g} \mathrm{mL}^{-1}$ zeocin (Invitrogen, USA) or $0.4 \mu \mathrm{g} \mathrm{mL}^{-1}$ norflurazon (Sigma-Aldrich, USA).

\section{Screening of transgenic strains}

Genomic DNA of native and transgenic $D$. tertiolecta cells was extracted using a modified Scott Newman's method (Newman et al. 1990) and used as a template for genomic PCR analysis to confirm the existence of transgenes. The gene-specific primers used for the detection of transformation plasmids are listed in Table 1. Each PCR reaction, consisting of $1 \mu \mathrm{g}$ genomic DNA, 
was set up according to the manufacturer's instructions (Thermo Fisher Scientific, USA). Amplification was performed at $95{ }^{\circ} \mathrm{C}$ for $5 \mathrm{~min}$ and cycled for 30 rounds of denaturation $\left(95^{\circ} \mathrm{C}\right.$ for $30 \mathrm{~s})$, annealing $\left(58^{\circ} \mathrm{C}\right.$ for $\left.30 \mathrm{~s}\right)$, and extension $\left(72{ }^{\circ} \mathrm{C}\right.$ for $\left.30 \mathrm{~s}\right)$, followed by a final extension $\left(72^{\circ} \mathrm{C}\right.$ for $\left.5 \mathrm{~min}\right)$.

\section{Real-time quantitative PCR}

Total RNA was extracted from native and transgenic D. tertiolecta cells with the RNeasy Plant Mini Kit (Qiagen, USA) and reverse transcribed to random-primed complementary DNA (cDNA) with the SuperScript III First-Strand Synthesis System (Invitrogen) according to the manufacturer's instructions. The real-time quantitative PCR (qPCR) was performed using a Bio-Rad CFX 96 Touch qPCR Detection System (Bio-Rad Laboratories) and 2× Maxima
SYBR Green/ROX qPCR Master Mix (Thermo Fisher Scientific) according to the manufacturer's instructions. Specific primers used for the analysis of gene expression are listed in Table 1. D. tertiolecta beta-tubulin gene, DtTUB, was used as an internal standard control. To normalize the relative gene expression across all samples, the starting amount of cDNA was standardized for all the samples.

\section{FA profiling and neutral lipid quantification}

The FA composition of lipids was analyzed using direct acidcatalyzed derivatization of FA methyl esters (FAMEs) as described previously (Lee et al. 2014) with minor modifications. The lyophilized sample was treated with $0.2 \mathrm{~mL}$ chloroform/ methanol $(2: 1, v / v)$ and $0.3 \mathrm{~mL}$ of $1.25 \mathrm{M}$ methanolic hydrochloride for $1 \mathrm{~h}$ at $85^{\circ} \mathrm{C}$. Tridecanoic acid (Sigma-Aldrich)

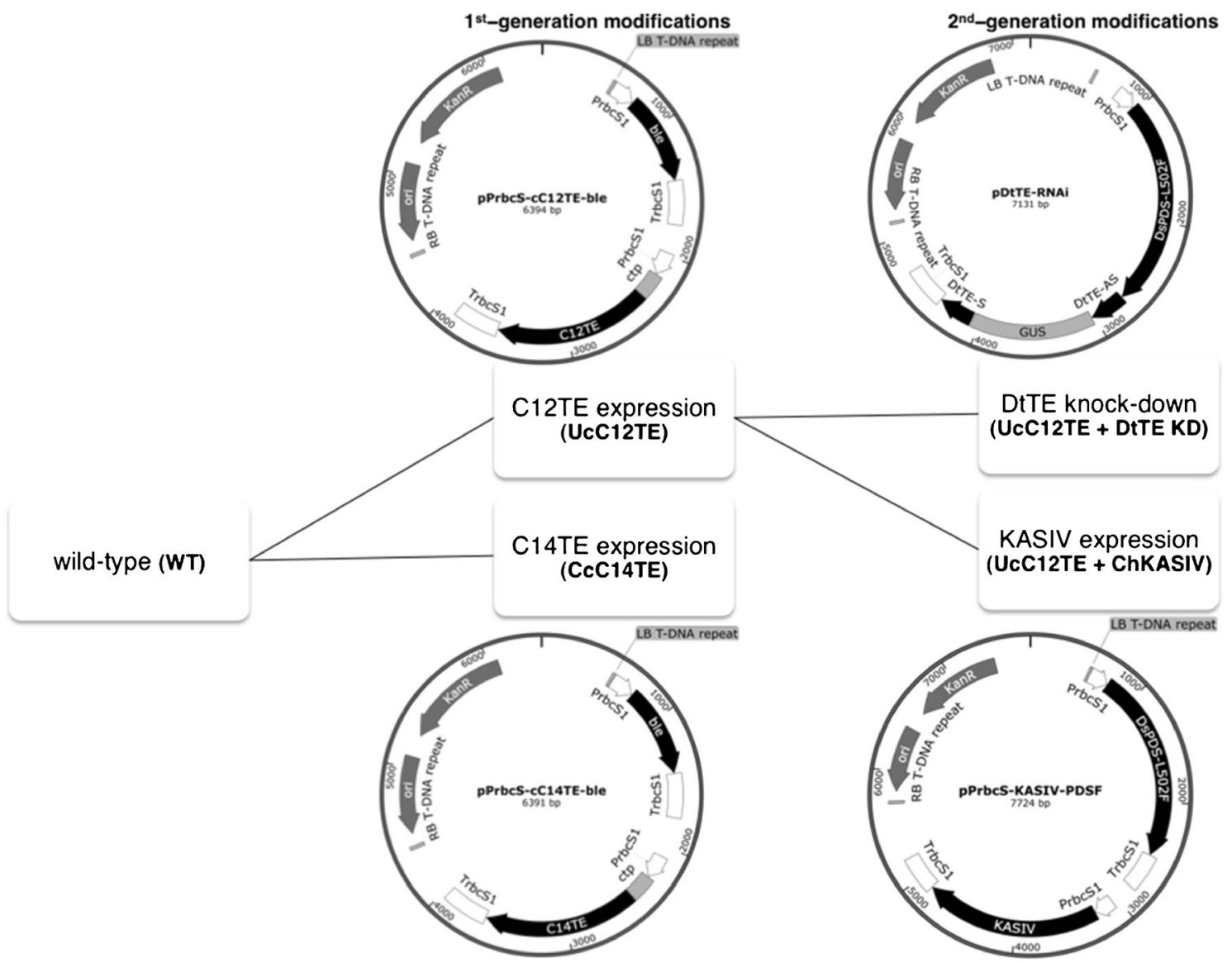

Fig. 1 Genetic engineering of $D$. tertiolecta to increase MCFA production. Sequential modifications are shown in flow chart, and detailed modifications are represented in schematic diagrams of transformation plasmids. As shown for pPrbcS-cC12TE-ble and pPrbcS-cC14TE-ble, $U$. californica C12:0-biased FatB TE (C12TE) and $C$. camphora C14:0-biased FatB TE (C14TE), together with chloroplast transit peptide (ctp), are respectively flanked with D. tertiolecta ribulose bisphosphate carboxylase small subunit 1 $($ DtrbcS1) promoter and terminator. Plasmid backbone contains kanamycin resistance cassette and positively selectable marker zeocin (ble) controlled by the DtrbcS1 promoter. In pDtTE-RNAi, the hairpin loop was generated using D. tertiolecta TE (DtTE) anti-sense (DtTE-AS) and sense $(D t T E-S)$ fragments linked with $\beta$-glucuronidase (GUS). In pPrbcS-KASIV-PDSF, Cuphea hookeriana MCFA-specific KAS $(K A S I V)$, together with ctp, are flanked with the DtrbcS1 promoter and terminator. Plasmid backbone contains kanamycin resistance cassette and positively selectable marker norflurazon (DSPDS-L502F) controlled by the DtrbcS1 promoter 
Table 1 List of primers used for detecting various genes in this study

\begin{tabular}{ll}
\hline Primer name & Primer sequence (5'-3') \\
\hline C12TE-GPCR-F & AGAAGCAGTGGACCAACCTG \\
C12TE-GPCR-R & AGCGGTGCTATCGTTGAACT \\
C14TE-GPCR-F & ACGGCACCAAGTTCAGCTAC \\
C14TE-GPCR-R & CTCCTGGTCCTGGTGTTCAT \\
DtTE-AS1-GPCR-F & ACGTGCTGGTGGCAGAACCA \\
DtTE-GUS-GPCR-R & TGCCATGTTCATCTGCCCAG \\
KASIV-GPCR-F & GCGAGATCAAGAGCTTCTCC \\
KASIV-GPCR-R & CTTGTTCAGCTCCTTCATGG \\
C12TE-RTF & AACCAGCACGTGAACAACAT \\
C12TE-RTR & GGTGCACTCCCTCCTGTACT \\
C14TE-RTF & GACGAGGAGATCAAGAAGCC \\
C14TE-RTR & ATGTTGTTCACGTGCTGGTT \\
DtTE-RTF & GGGACATGGTCACAGTTGAG \\
DtTE-RTR & GGTGGCAGAACCATACTCCT \\
KASIV-RTF & GCGAGATCAAGAGCTTCTCC \\
KASIV-RTR & CTTGTTCAGCTCCTTCATGG \\
DtTUB-RTF & CAGATGTGGGATGCCAAGAACAT \\
DtTUB-RTR & GTTCAGCATCTGCTCATCCACCT \\
\hline
\end{tabular}

was included to correct for the loss of FAMEs from incomplete derivatization and subsequent extraction. Then, $1 \mathrm{~mL}$ hexane was used to extract the derivatized FAMEs for $1 \mathrm{~h}$ at room temperature. Extracted FAMEs were analyzed using an Agilent 7890B gas chromatograph (GC) (Agilent Technologies, USA) equipped with an Agilent 5977A electron ionization mass spectrometric detector (MS). The GC inlet temperature was set to $240{ }^{\circ} \mathrm{C}$ with an injection volume of $1.0 \mu \mathrm{L}$ and a 5:1 split ratio. Helium was used as the carrier gas in a constant flow rate at $1.0 \mathrm{~mL} \mathrm{~min}^{-1}$. A highly polar stationary phase-fused silica capillary column of cyanopropyl polysiloxane with a film thickness dimension of $100 \mathrm{~m} \times 0.25 \mathrm{~mm} \times 0.20 \mu \mathrm{m}(\mathrm{CP}-\mathrm{Sil} 88$; Agilent Technologies) was used for analysis. The GC parameters were programmed as follows: the initial oven temperature of $80^{\circ} \mathrm{C}$ was held for $3 \mathrm{~min}$ before being ramped at $4{ }^{\circ} \mathrm{C} \min ^{-1}$ to $220{ }^{\circ} \mathrm{C}$ for $5 \mathrm{~min}$ and at $4{ }^{\circ} \mathrm{C} \mathrm{min}{ }^{-1}$ to $240{ }^{\circ} \mathrm{C}$ for $10 \mathrm{~min}$. The total run time was $55 \mathrm{~min}$. The MS parameters were set as follows: the mass range was 40 to $500 \mathrm{Da}$ in full scan mode, and the auxiliary, MS source, and MS quad temperatures were set to 240,230 , and $150{ }^{\circ} \mathrm{C}$, respectively. Quantification of FAMEs was performed using the MassHunter Workstation software (Agilent Technologies) against a series of C4-C24 FAME standards (Sigma-Aldrich). The FA composition in $D$. tertiolecta indicated the percentage composition of each FA obtained by dividing the quantity of each FA by the sum of total FAs normalized to sample dry weight and FAME recovery. Neutral lipids were quantified by a Nile red staining method (Tan et al. 2016).

\section{Results and discussion}

\section{Generation of MCFA producing D. tertiolecta strains}

The primary aim of this study was to investigate whether the availability of fatty acyl-ACP substrate pool for TE activity is one factor that limits the accumulation of MCFAs in transgenic D. tertiolecta strains. We hypothesize that (1) there is a competition between exogenous and endogenous TEs and (2) KAS is capable of distributing different chain lengths of fatty acyl-ACPs. We constructed two generations of D. tertiolecta strains for MCFA production (Fig. 1). All of the strains were carefully segregated to ensure their genotypic and phenotypic purities. In the first-generation $D$. tertiolecta strains (UcC12TE and CcC14TE), we selected C12TE and C14TE, from U. californica and C. camphora, respectively, based on their abilities to produce MCFAs (Radakovits et al. 2011). These genes were cloned in the well-characterized pGreenII 0000 plasmid (Hellens et al. 2000) and were separately transformed into wild-type $D$. tertiolecta, under the selection of zeocin resistance. We also included the chloroplast transit peptide from DtSBPase to ensure that the transgenic TE protein was directed to the site of de novo FA synthesis in the chloroplast. Multiple independent zeocin ${ }^{\mathrm{r}}$ colonies were obtained and screened by genomic PCR. Figure $2 a, b$ shows the genomic PCR gel images of the successful amplification of partial fragments of C12TE and C14TE from some of the transformed strains. Specifically, a $0.5-\mathrm{kb}$ amplified product was detected, using either C12TE or C14TE gene-specific primers. As expected, exogenous $\mathrm{C} 12 \mathrm{TE}$ and $\mathrm{C} 14 \mathrm{TE}$ were not detected in wild-type $D$. tertiolecta. C12TE and C14TE transcript levels of the transgenic strains were determined by real-time qPCR (Fig. 3a, b). All transgenic strains revealed varying transgene expression levels, which might possibly be due to epigenetic silencing effects of transgenes or different numbers/locations of integrated transgene copies (Cerutti et al. 1997; Wu-Scharf et al. 2000).

For the second-generation D. tertiolecta strain (UcC12TE + DtTE KD), we suppressed the expression of the DtTE gene by RNA interference (RNAi). This was performed to investigate the competitive nature of endogenous TE in a MCFA-producing strain of D. tertiolecta. An RNAi plasmid was transformed into mutant cC12TE_A4 D. tertiolecta (from UcC12TE background) under the selection of norflurazon resistance. Endogenous TE could not be completely knocked out because some LCFAs are required to synthesize essential membrane lipids (Chapman and Ohlrogge 2012). Several norflurazon ${ }^{r}$ colonies were isolated for genomic PCR screening. An expected $0.5-\mathrm{kb}$ amplified product using gene-specific DtTE-AS sense primer and GUS antisense primer was obtained in the double C12TE overexpressed and DtTE knockdown mutants (Fig. 2c). The 26 positive transformants from genomic PCR results were 
(a)

UcC12TE

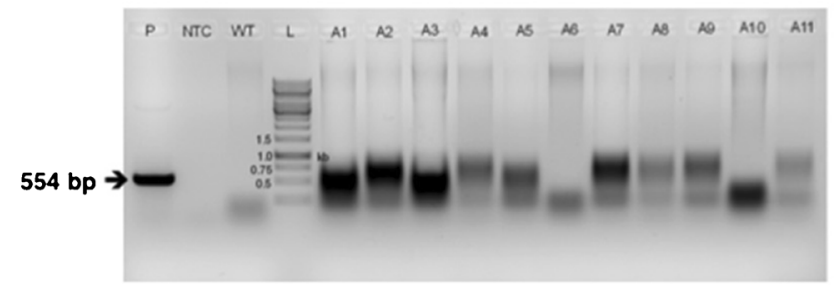

(b)

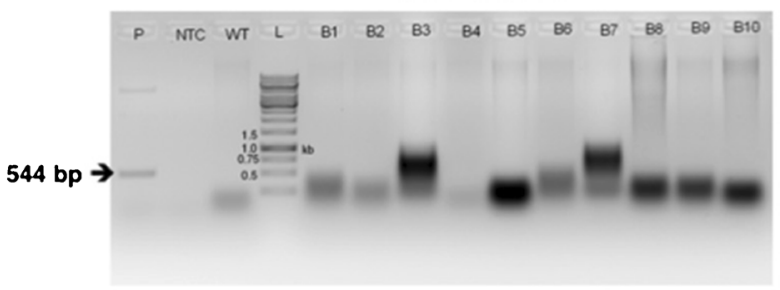

(d)

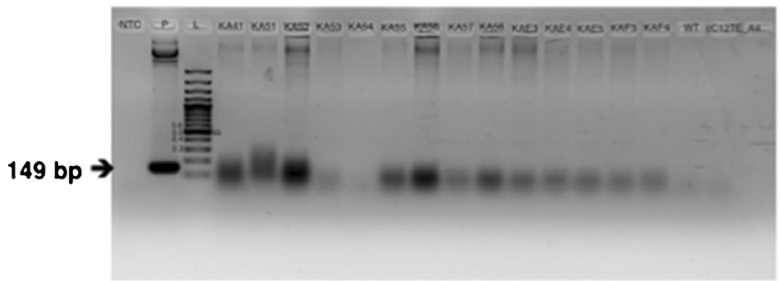

Fig. 2 Genomic PCR identification of MCFA producing D. tertiolecta strains. Amplification of the partial fragments of transgenes from the genomic DNA of mutant and wild-type $D$. tertiolecta cells using genespecific primers. $P$ plasmid, NTC non-template control, WT genomic DNA wild-type, $L$ 1-kb ladder marker. a Genomic DNA pPrbcS-

analyzed for the relative DtTE gene expression levels by realtime qPCR. Out of the 26 norflurazon $^{\mathrm{r}}$ colonies, 8 showed reduced DtTE gene expression by about $50 \%$, while 11 showed reduced DtTE gene expression by about $20 \%$ with respect to the mutant cC12TE_A4 and wild-type D. tertiolecta (Fig. 3c). This reduction of DtTE expression was, however, far from the previously reported $A$. thaliana FatB TE knockout mutant, which had displayed 150-fold lower in expression than that of control plant (Bonaventure et al. 2003). Therefore, UcC12TE + DtTE KD mutants were considered as strains deficient in DtTE gene expression but not a total loss of function.

For the second-generation of $D$. tertiolecta strain (UcC12TE + ChKASIV), to allow a fair comparison with the strain UcC12TE + DtTE KD, KASIV from C. hookeriana was transformed into mutant cC12TE A4 $D$. tertiolecta (from UcC12TE background). Studies on seed oil profiles in the Arabidopsis plants overexpressing both Cuphea KASIV and FatB TE reported a reduction in (c)

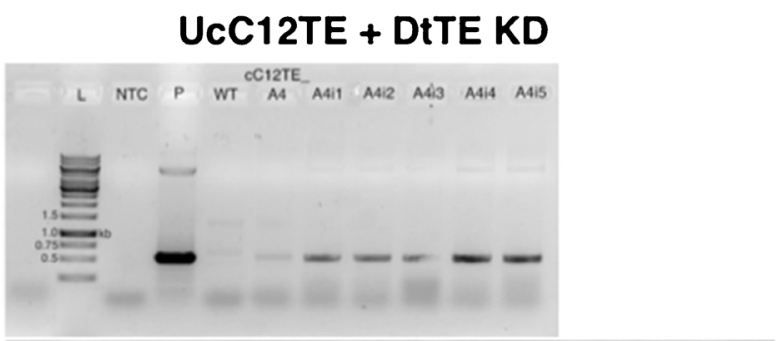

N

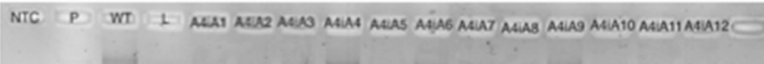

550 bp $\rightarrow$
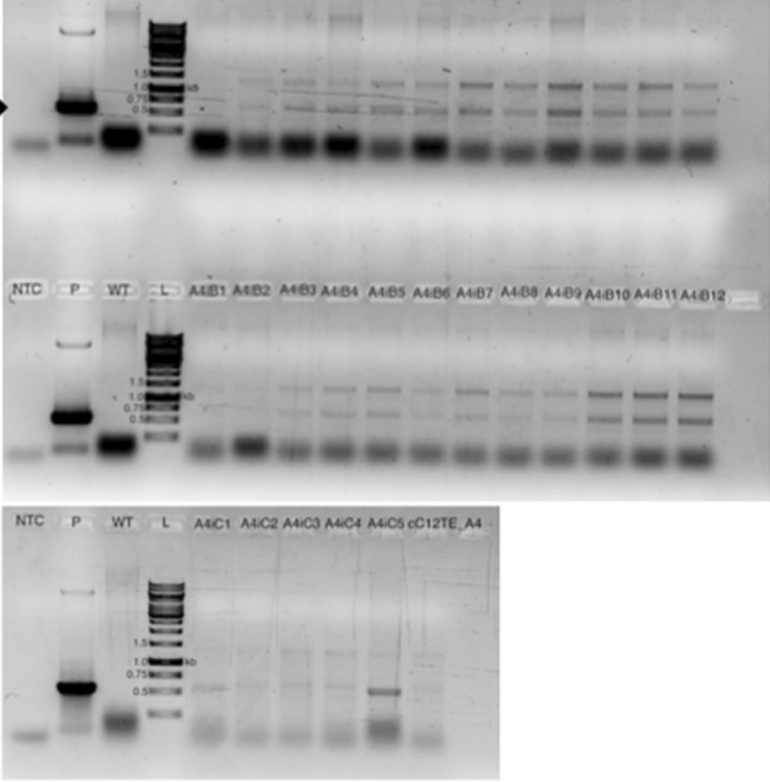

cC12TE-ble mutants. b Genomic DNA pPrbcS-cC14TE-ble mutants. c Genomic DNA pDtTE-RNAi mutants. d Genomic DNA pPrbcS-KASIVPDSF mutants. UcC12TE U. californica C12:0-biased FatB TE, CcC14TE C. camphora C14:0-biased FatB TE, DtTE KD D. tertiolecta TE knockdown, ChKASIV C. hookeriana MCFA-specific KAS

the levels of C16:0 and an enhancement in the levels of C12:0 (Leonard et al. 1998). The overexpression of KASIV in a MCFA-producing strain of D. tertiolecta would allow us to examine whether the bottleneck of further MCFA accumulation lies in the control of a KAS enzyme to generate MCFA acyl-ACP substrates. Several norflurazon ${ }^{\mathrm{r}}$ colonies were isolated, and the presence of KASIV was verified by genomic PCR, using the KASIV gene-specific primers (Fig. 2d). As expected, exogenous KASIV could not be detected in mutant cC12TE_A4 and wild-type D. tertiolecta. Additionally, we only confirmed the expression of the KASIV gene in one transgenic strain (Fig. 3d).

\section{Co-expression of C12TE and KASIV increased MCFA production by fourfold}

Production of storage lipids in D. tertiolecta is usually low during the exponential growth phase and increases during 
(a)

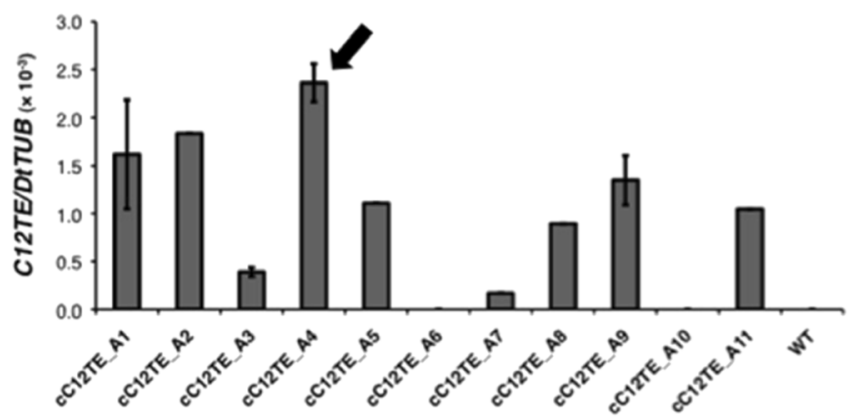

(c)

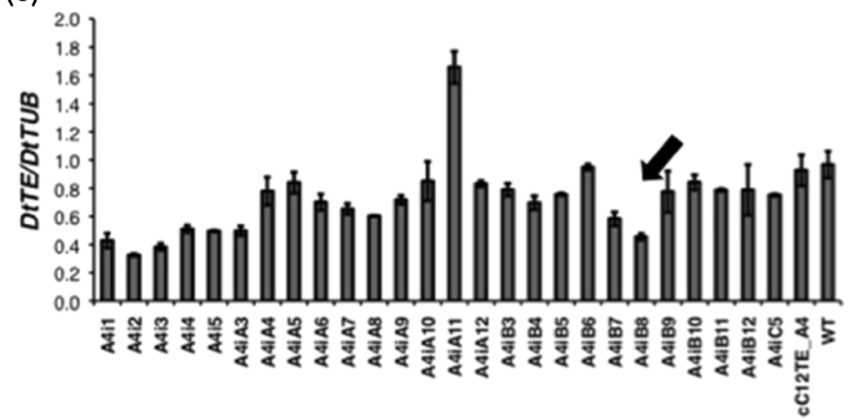

Fig. 3 Transcript abundance analysis of native and transgenic $D$. tertiolecta strains. Gene expression detected by real-time qPCR in strains a UcC12TE, b CcC14TE, c UcC12TE + DtTE KD, and d UcC12TE + ChKASIV. Arrows indicate the transgenic D. tertiolecta

the stationary phase (Tan et al. 2016). Therefore, all strains were harvested at the stationary phase for higher detection sensitivity, and MCFA levels were determined by GC-MS. Increments of MCFA amounts in transgenic D. tertiolecta strains were then represented in fold changes with respect to the wild-type $D$. tertiolecta MCFA amounts (Fig. 4). Except for strain UcC12TE + DtTE KD, most genetic modifications had resulted in significant increments of MCFA production compared with the wild-type D. tertiolecta. In strain $\mathrm{UcC} 12 \mathrm{TE}$ and $\mathrm{CcC} 14 \mathrm{TE}$, expression of single $\mathrm{C} 12 \mathrm{TE}$ and C14TE, respectively, resulted in almost onefold increased total MCFA production (Fig. 4a), with strain Cc14TE giving the greatest response of fourfold increased C12:0 accumulation (Fig. 4b). Unexpectedly, in strain Cc14TE, there was barely an increase in C14:0 accumulation (Fig. 4c). MCFA production was further enhanced by co-expression of KASIV. In strain UcC12TE + ChKASIV, there was fourfold increased MCFA accumulation (Fig. 4a). Levels of C12:0 and C14:0 respectively increased by sevenfold and threefold (Fig. 4b, c).

We have successfully increased accumulation of MCFAs in those transgenic $D$. tertiolecta strains constructed by the single overexpression of C12TE or C14TE and also the double overexpression of C12TE and KASIV. However, our observations were in contrast to a previous report by Blatti and colleagues (2012). They did not observe a significant increase in the (b)

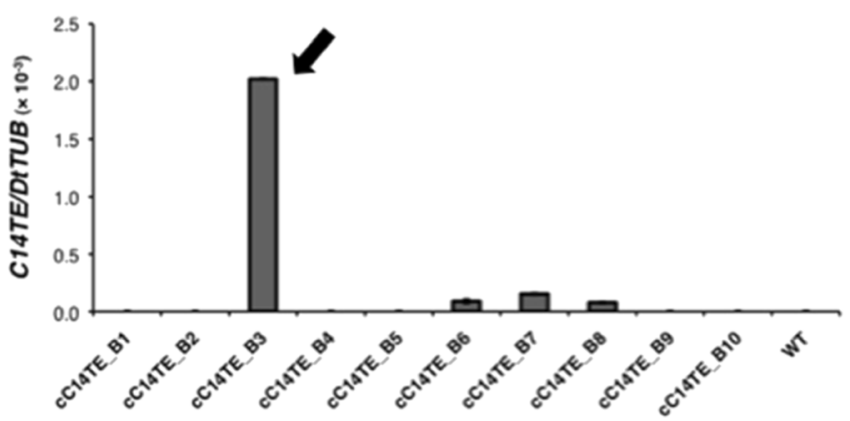

(d)

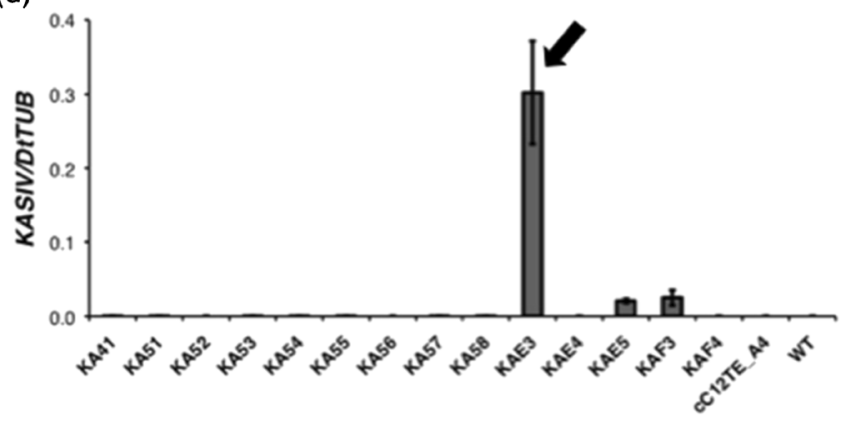

strains selected for further phenotypic characterization. Values are the average of three separate experiments, and error bars show the standard deviation

MCFA levels after introducing the C12:0-biased FatB TE from $U$. californica alone, because of the weak proteinprotein interaction between the exogenous TE and the endogenous acyl-ACP. Our results showed that the single introduction of MCFA-specific TE (C12TE or C14TE) could actually increase the production of MCFAs. This disparity may be explained by the location of transgene deposition in their respective genomes and the method of MCFA analysis. Blatti and colleagues analyzed MCFA levels in transgenic C. reinhardtii strains during the exponential growth phase. On the other hand, we analyzed MCFA levels at the stationary phase to induce TAG production. Few studies of native Chaetoceros strain GSL56 (Gu et al. 2016), transgenic strains of $C$. reinhardtii (Inaba et al. 2017), and P. tricornutum (Radakovits et al. 2011) revealed that majority of MCFAs produced were preferentially incorporated into TAGs. It may be more effective to detect minor differences in MCFA amounts between the native and transgenic strains under our harvesting conditions. Nonetheless, the increment we observed was modest, and more works would be needed to further optimize the accumulation of MCFAs.

For our purpose of improving MCFA synthesis in $D$. tertiolecta, the double genetic modification approach, involving co-expression of C12TE and KASIV, performs better than the traditional approach of a single introduction of 
(a)

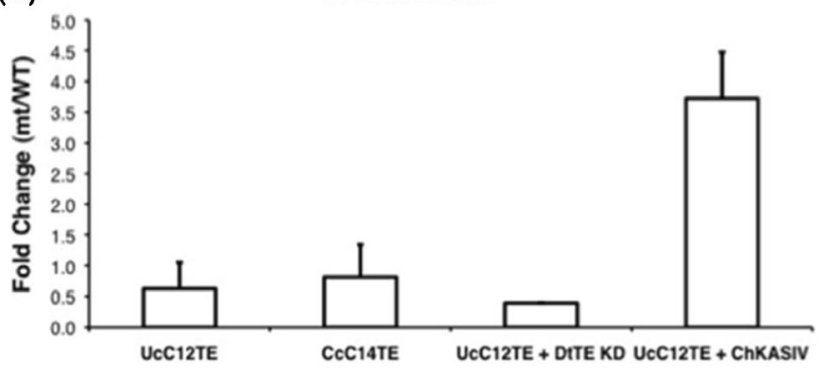

(b)

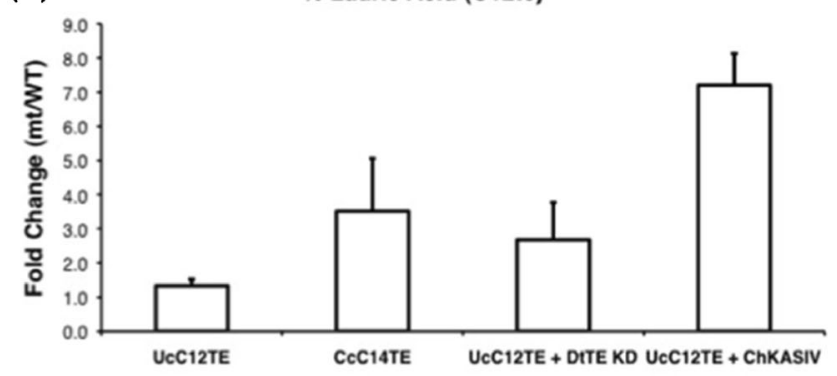

(c)

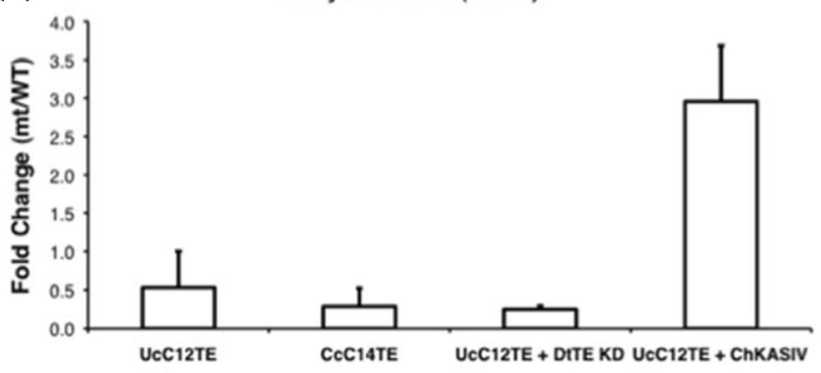

Fig. 4 Enhanced MCFA production in transgenic D. tertiolecta strains. The increments in the a percentages of total MCFAs, $\mathbf{b}$ percentages of lauric acid (C12:0), and $\mathbf{c}$ percentages of myristic acid (C14:0) are expressed as fold changes (mutant/wild-type). Values are the average of three separate experiments, and error bars show the standard deviation. UcC12TE U. californica C12:0-biased FatB TE, CcC14TE C. camphora C14:0-biased FatB TE, DtTE KD D. tertiolecta TE knockdown, ChKASIV C. hookeriana MCFA-specific KAS

MCFA-specific TE and the silencing of endogenous DtTE. The progressive increases in C12:0 and C14:0 accumulation in strain UcC12TE + ChKASIV demonstrated that C12TE and KASIV enzymes both contribute to the regulation of FA chain lengths in D. tertiolecta. This approach can be employed to increase MCFA composition of the microalgae oil. Enhanced MCFA production in our double transgenic $D$. tertiolecta strain may be due to (1) enlargement of the MCFA acyl-ACP substrate pool by overexpressing KASIV (Dehesh et al. 1998), (2) improvement to an overall FA synthase rate that matches a TE activity (Dehesh et al. 2001), and/ or (3) concentration of MCFA acyl-ACP substrate pool caused by KAS enzyme (Abbadi et al. 2000). On the other hand, the lack of significant DtTE knockdown impact on the production of MCFAs in transgenic D. tertiolecta strain suggested that endogenous DtTE was not a major controller of MCFA acylACP substrate pool.

\section{Enhanced MCFA accumulation had negative impact on total lipid production in transgenic $D$. tertiolecta strains}

Microalgae synthesize and store different kinds of lipids in a single cell (Hu et al. 2008). In contrast to the conventional FA compositions of microalgae that contain LCFAs from C16 to C18, MCFAs (from C8 to C14) are generally classified to be unusual. It is possible that an increased production of MCFAs would potentially have deleterious effects on the cells. Nile red assay revealed that the mutants accumulated half the amounts of neutral lipids than wild-type $D$. tertiolecta (Fig. $5 \mathrm{a})$. The decreased production of neutral lipids could be the result of decreased synthesis of total lipids. Two strains, namely CcC14TE and UcC12TE + ChKASIV, had displayed almost threefold reduction of total lipids (Fig. 5b). It is surprisingly to note that these strains were also the highest MCFA producers, in particular for C12:0 accumulation (Fig. 4b). The observation could suggest physiological responses of transgenic D. tertiolecta strains towards heightened levels of unusual MCFAs. Nonetheless, we also could not exclude that the decreased production of total lipids was due to secondary mutations.

Our observation here opposed to the previous reports of higher lipid production and free FA secretion in bacterial and cyanobacterial systems after the overexpression of MCFA-specific TEs (Voelker and Davies 1994; Lu et al. 2008). In those reports, the increment of total lipid production was dependent on the simultaneous overexpression of TEs with the inactivation of FA catabolic pathways. Furthermore, eukaryotic microalgae (D. tertiolecta) and bacteria/cyanobacteria are innately different, given that eukaryotic microalgae are capable to store free FAs in the form of storage TAGs. This could explain the reason for not able to detect any free FA secretion from $D$. tertiolecta cells, and this was also supported by other FA secretion works done on eukaryotic microalgae (Radakovits et al. 2011; Tan and Lee 2017).

Some differences in FA profiles of transgenic D. tertiolecta strains were also observed (Fig. 5c). Transgenic D. tertiolecta strains exhibited an increment in very long-chain-length FAs (vLCFAs) and a slight reduction in $\mathrm{C} 16: 0$ and $\mathrm{C} 18: 3$ that are the major FA species types in D. tertiolecta (Chen et al. 2011). This could be explained by the additional TEs in the transgenic strains, which have conferred substrate preference for saturated acyl-ACPs. Nevertheless, increased accumulation of vLCFAs may also reflect a change in the transgenic strain membrane properties. The mechanism that edits out unusual MCFAs from membrane lipids and channels them to 
(a)

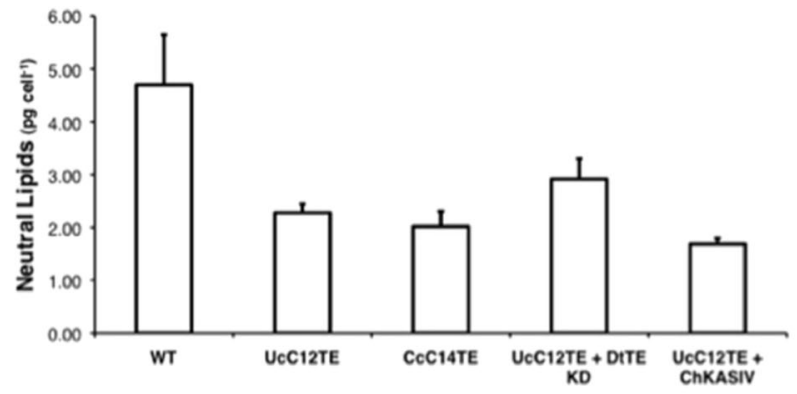

(b)

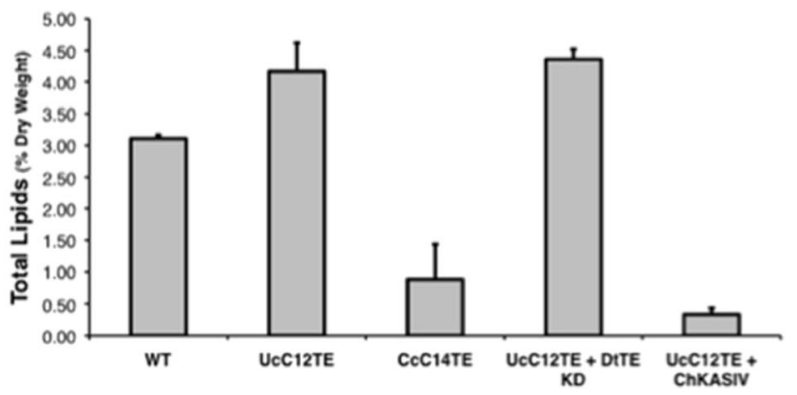

(c)
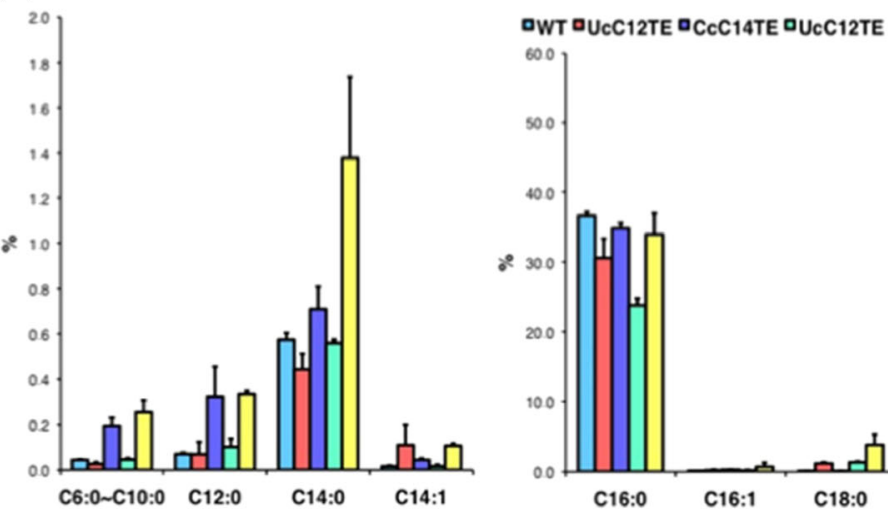

Fig. 5 Quantitative analyses of neutral lipids, total lipids, and FA profiles. a Neutral lipids were estimated using Nile red assay. b Total lipids per dry weight were measured by GC-MS. c FA compositions were calculated based on weight percentages $(\%)$. Values are the

proper storage lipids is imperative for unicellular microalgae. It is believed that MCFA would affect the structural integrity of membranes and have detrimental effects on the cells (Millar et al. 2000). Therefore, mechanisms that regulate MCFA accumulation are not solely govern by the pool of MCFA acyl-ACP substrates but also how the transgenic strains cope with the unusual MCFA buildup in the cells.

\section{Conclusion}

Our results showed that the double-genetic engineering strategy of employing chain-length specific TE and KAS significantly enhanced MCFA composition of $D$. tertiolecta oil. This study illustrates that enlarging MCFA acyl-ACP substrate pool is one feasible approach that may be useful in conjunction to other strategies to sustainably produce good quality of microalgae-based biodiesel containing high levels of MCFAs. While transgenic TE and KAS are able to redirect FA synthesis from long to medium chain lengths, the increases in yield are still not as high as those achieved in plants and bacteria. Future efforts to understand microalgal FA homeostasis are necessary, whether targeting MCFA productivity or other unusual FA chain lengths.
Acknowledgements This work was supported by the National Research Foundation (NRF), Prime Minister's Office, Singapore, under the Campus for Research Excellence and Technological Enterprise (CREATE) Program (grant number R-182-000-205-592).

\section{Compliance with ethical standards}

Conflict of interest The authors declare that they have no conflict of interest.

Open Access This article is distributed under the terms of the Creative Commons Attribution 4.0 International License (http:// creativecommons.org/licenses/by/4.0/), which permits unrestricted use, distribution, and reproduction in any medium, provided you give appropriate credit to the original author(s) and the source, provide a link to the Creative Commons license, and indicate if changes were made.

\section{References}

Abbadi A, Brummel M, Spener F (2000) Knockout of the regulatory site of 3-ketoacyl-ACP synthase III enhances short- and medium-chain acyl-ACP synthesis. Plant J 24:1-9

Blatti JL, Beld J, Behnke CA, Mendez M, Mayfield SP, Burkart MD (2012) Manipulating fatty acid biosynthesis in microalgae for biofuel through protein-protein interactions. PLoS One 7(9):e42949

Bonaventure G, Salas JJ, Pollard MR, Ohlrogge JB (2003) Disruption of the FATB gene in Arabidopsis demonstrates an essential role of saturated fatty acids in plant growth. Plant Cell 15:1020-1033 
Cerutti H, Johnson AM, Gillham NW, Boynton JE (1997) Epigenetic silencing of a foreign gene in nuclear transformants of Chlamydomonas. Plant Cell 9(6):925-945

Chapman KD, Ohlrogge JB (2012) Compartmentation of triacylglycerol accumulation in plants. J Biol Chem 287:2288-2294

Chen M, Tang H, Ma H, Holland TC, Ng KY, Salley SO (2011) Effect of nutrients on growth and lipid accumulation in the green algae Dunaliella tertiolecta. Bioresour Technol 102:1649-1655

Davies HM, Anderson L, Fan C, Hawkins DJ (1991) Developmental induction, purification, and further characterization of 12:0-ACP thioesterase from immature cotyledons of Umbellularia californica. Arch Biochem Biophys 290:37-45

Dehesh K (2001) How can we genetically engineer oilseed crops to produce high levels of medium-chain fatty acids? Eur J Lipid Sci Technol 103:688-697

Dehesh K, Edwards P, Fillatti J, Slabaugh M, Byrne J (1998) KAS IV: a 3-ketoacyl-ACP synthase from Cuphea sp. is a medium chain specific condensing enzyme. Plant J 15:383-390

Dehesh K, Jones A, Knutzon DS, Voelker TA (1996) Production of high levels of 8: 0 and 10: 0 fatty acids in transgenic canola by overexpression of Ch FatB2, a thioesterase cDNA from Cuphea hookeriana. Plant J 9:167-172

Dehesh K, Tai H, Edwards P, Byrne J, Jaworski JG (2001) Overexpression of 3-ketoacyl-acyl-carrier protein synthase IIIs in plants reduces the rate of lipid synthesis. Plant Physiol 125:1103-1114

Durrett TP, Benning C, Ohlrogge J (2008) Plant triacylglycerols as feedstocks for the production of biofuels. Plant J 54:593-607

Gong Y, Guo X, Wan X, Liang Z, Jiang M (2011) Characterization of a novel thioesterase (PtTE) from Phaeodactylum tricornutum. J Basic Microbiol 51:666-672

Gu H, Jinkerson RE, Davies FK, Sisson LA, Schneider PE, Posewitz MC (2016) Modulation of medium-chain fatty acid synthesis in Synechococcus sp. PCC 7002 by replacing FabH with a Chaetoceros ketoacyl-ACP synthase. Front Plant Sci 7(690). doi: 10.3389/fpls.2016.00690

Hellens RP, Edwards EA, Leyland NR, Bean S, Mullineaux PM (2000) pGreen: a versatile and flexible binary Ti vector for Agrobacteriummediated plant transformation. Plant Mol Biol 42:819-832

$\mathrm{Hu} \mathrm{Q}$, Sommerfeld M, Jarvis E, Ghirardi M, Posewitz M, Seibert M, Darzins A (2008) Microalgal triacylglycerols as feedstocks for biofuel production: perspectives and advances. Plant J 54:621-639

Inaba Y, Nakahigashi K, Ito T, Tomita M (2017) Alteration of fatty acid chain length of Chlamydomonas reinhardtii by simultaneous expression of medium-chain-specific thioesterase and acyl carrier protein. Phycol Res 65:94-99

Jing F, Cantu DC, Tvaruzkova J, Chipman JP, Nikolau BJ, YandeauNelson MD, Reilly PJ (2011) Phylogenetic and experimental characterization of an acyl-ACP thioesterase family reveals significant diversity in enzymatic specificity and activity. BMC Biochem 12:44

Knothe G, Van Gerpen JH, Krahl J (2005) The biodiesel handbook. AOCS

Lee S-Y, Kim S-H, Hyun S-H, Suh HW, Hong S-J, Cho B-K, Lee C-G, Lee H, Choi H-K (2014) Fatty acids and global metabolites profiling of Dunaliella tertiolecta by shifting culture conditions to nitrate deficiency and high light at different growth phases. Process Biochem 49:996-1004

Leonard JM, Knapp SJ, Slabaugh MB (1998) A Cuphea beta-ketoacylACP synthase shifts the synthesis of fatty acids towards shorter chains in Arabidopsis seeds expressing Cuphea FatB thioesterases. Plant J 13:621-628

Lin H, Fang L, Low CS, Chow Y, Lee YK (2013) Occurrence of glycerol uptake in Dunaliella tertiolecta under hyperosmotic stress. FEBS J 280:1064-1072

Liu J, Gerken H, Huang J, Chen F (2013) Engineering of an endogenous phytoene desaturase gene as a dominant selectable marker for Chlamydomonas reinhardtii transformation and enhanced biosynthesis of carotenoids. Process Biochem 48:788-795

Lu X, Vora H, Khosla C (2008) Overproduction of free fatty acids in E. coli: implications for biodiesel production. Metab Eng 10:333-339

Millar AA, Smith MA, Kunst L (2000) All fatty acids are not equal: discrimination in plant membrane lipids. Trends Plant Sci 5:95-101

Newman SM, Boynton JE, Gillham NW, Randolph-Anderson BL, Johnson AM, Harris EH (1990) Transformation of chloroplast ribosomal RNA genes in Chlamydomonas: molecular and genetic characterization of integration events. Genetics 126:875-888

Radakovits R, Eduafo PM, Posewitz MC (2011) Genetic engineering of fatty acid chain length in Phaeodactylum tricornutum. Metab Eng 13:89-95

Stansell GR, Gray VM, Sym SD (2012) Microalgal fatty acid composition: implications for biodiesel quality. J Appl Phycol 24:791-801

Stevens DR, Rochaix JD, Purton S (1996) The bacterial phleomycin resistance gene ble as a dominant selectable marker in Chlamydomonas. Mol Gen Genet 251:23-30

Tan KWM, Lee YK (2017) Expression of the heterologous Dunaliella tertiolecta fatty acyl-ACP thioesterase leads to increased lipid production in Chlamydomonas reinhardtii. J Biotech 247:60-67

Tan KWM, Lin H, Shen H, Lee YK (2016) Nitrogen-induced metabolic changes and molecular determinants of carbon allocation in Dunaliella tertiolecta. Scientific Rep 6:37235. doi:10.1038/ srep37235 http://www.nature.com/articles/srep37235\# supplementary-information

Tang H, Abunasser N, Garcia MED, Chen M, Simon Ng KY, Salley SO (2011) Potential of microalgae oil from Dunaliella tertiolecta as a feedstock for biodiesel. Appl Energy 88:3324-3330

Thelen JJ, Ohlrogge JB (2002) Metabolic engineering of fatty acid biosynthesis in plants. Metab Eng 4:12-21

Voelker T, Kinney AJ (2001) Variations in the biosynthesis of seedstorage lipids. Annu Rev Plant Physiol Plant Mol Biol 52:335-361

Voelker TA, Davies HM (1994) Alteration of the specificity and regulation of fatty acid synthesis of Escherichia coli by expression of a plant medium-chain acyl-acyl carrier protein thioesterase. J Bacteriol 176:7320-7327

Voelker TA, Worrell AC, Anderson L, Bleibaum J, Fan C, Hawkins DJ, Radke SE, Davies HM (1992) Fatty acid biosynthesis redirected to medium chains in transgenic oilseed plants. Science 257:72-74

Walker TL, Becker DK, Collet C (2005) Characterisation of the Dunaliella tertiolecta $R b c S$ genes and their promoter activity in Chlamydomonas reinhardtii. Plant Cell Rep 23:727-735

Wu-Scharf D, Jeong B, Zhang C, Cerutti H (2000) Transgene and transposon silencing in Chlamydomonas reinhardtii by a DEAH-box RNA helicase. Science 290:1159-1162

Zinoviev S, Muller-Langer F, Das P, Bertero N, Fornasiero P, Kaltschmitt M, Centi G, Miertus S (2010) Next-generation biofuels: survey of emerging technologies and sustainability issues. ChemSusChem 3: 1106-1133 